\title{
DISSEMINATED NOCARDIOSIS IN AN ELDERLY PATIENT PRESENTING WITH PROLONGED PYREXIA: DIAGNOSIS BY THYROID ABSCESS CULTURE
}

\author{
*VA Indumathi, NS Shivakumar
}

\begin{abstract}
We report a case of nocardiosis in an immunosuppressed elderly patient who presented with prolonged pyrexia. Nocardia asteroides was isolated from the thyroid, with CT scan evidence of dissemination to the brain, abdomen and lungs. The patient succumbed to illness despite aggressive therapy. Autopsy could not be performed. To the best of our knowledge, this is the first reported case from India, on Nocardia asteroides affecting the thyroid tissue.
\end{abstract}

Key words: Dissemination, Nocardia asteroides, thyroid abscess

Nocardia asteroides complex is an important opportunistic agent in immunosuppressed patients. Nocardia are classically aerobic, gram positive, filamentous, weakly acid-fast bacilli, ubiquitous soil saprophytes that cause opportunistic exogenous infection. Usually primary pulmonary infection occurs followed by dissemination. As described in the literature, almost every organ can be infected. ${ }^{1}$ However, infection of thyroid glands is rare. To our knowledge, only five cases of Nocardia isolated from thyroid tissue have been published. ${ }^{1-}$ ${ }^{5}$ Nocardia infection is difficult to diagnose by clinical or radiological methods because of its protean manifestations. 6 We report here a case of thyroid abscess caused by Nocardia asteroides.

\section{Case Report}

A 67-year-old male, retired forest officer, was admitted with complaints of fever and headache for 10 days, cough with haemopytsis for three days, abdomen pain for three days, reduced urine output for three days and altered sensorium for one day.

The patient was a known diabetic on insulin over the last 25 years. He was extensively evaluated for pyrexia of uncertain origin during the last one year and was on regular steroids, being diagnosed as adult Still's disease/vasculitis.

Physical examination showed that he was disoriented, febrile, tachypnoeic, with stable vitals, bilateral basal crepitations, hepatosplenomegaly, extensive purpuric rashes on the chest, abdomen and the limbs and a swelling in the thyroid region. A provisional diagnosis of pneumonia with sepsis was made and was started on meropenem and amikacin.

*Corresponding author (email: <indumathi222000@rediffmail.com>) Gokula Metropolis Clinical Laboratory (VAI) and Department of Medicine (NSS), MS Ramaiah Medical Teaching Hospital, Bangalore - 560 054, Karnataka, India

Received: 28-12-06

Accepted: 15-02-07
Radiological investigations revealed mixed solid and cystic mass in the head of the pancreas, large omentum (Fig. 1), multiple focal hypodense lesion in the brain (Fig. 2) bilateral pulmonary nodules with cavitation, mediastinal and hilar nodules and a mass in the left thyroid lobe as evidenced by the computed tomography.

Laboratory investigations showed neutrophilic leukocytosis, mildly elevated liver function test; cerebrospinal fluid (CSF) analysis had a cell count of $535 / \mu \mathrm{L}$ predominantly polymorphs, normal sugar levels and elevated protein. Blood, CSF, urine and endotracheal secretion cultures did not yield any significant findings.

Based on clinical and radiological findings that strongly

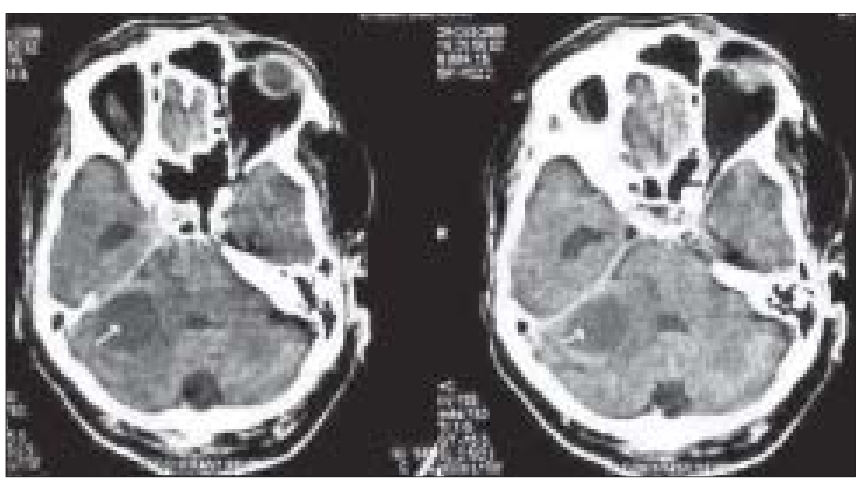

Figure 1: CT brain showing an abscess in the right posterior cranium

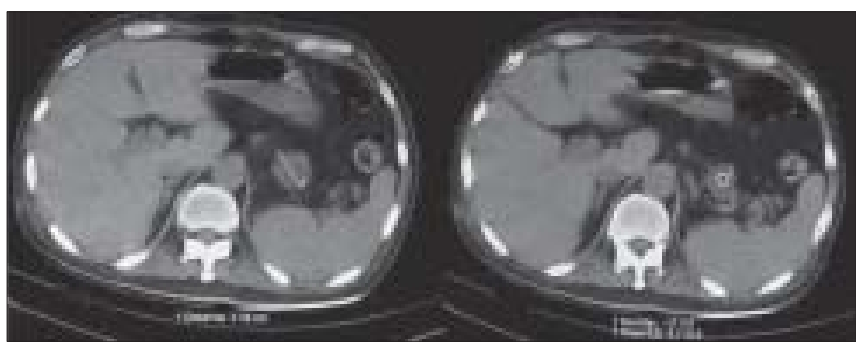

Figure 2: CT Abdomen showing hypodensity in the head of the pancreas 
suggested neoplasia and in view of thyroid swelling, a fine needle aspiration (FNAC) was performed for malignant cells. FNAC showed a purulent material with no malignant cells. However, there were filamentous structures strongly suggestive of an infective cause. Therefore, the fine needle aspiration was repeated for culture and Gram stain. Gram stained smear showed gram variable filamentous organisms strongly suggestive of Nocardia and the modified ZN stain $\left(1 \% \mathrm{H}_{2} \mathrm{SO}_{4}\right.$ showed acid fast branching filamentous organisms that resembled Nocardia (Fig. 3).

Based on the smear findings and awaiting culture confirmation, the patient was started on cotrimoxazole. The specimen was cultured on chocolate agar, blood agar, Sabouraud dextrose agar and Lowenstein Jensen (LJ) medium. After 72 hours, dull, rough, wrinkled colonies appeared on all media. We identified the isolate as Nocardia by the following biochemical tests: catalase: positive; urease: hydrolysed; oxidase: negative; aesculin: hydrolysed; nitrate: reduced. ${ }^{7}$ Antibiotic susceptibility was performed by the Kirby Bauer's disc diffusion method. ${ }^{7}$ The isolate was found to be resistant to penicillin, erythromycin and ampicillin and sensitive to cotrimoxazole, amikacin, cefotaxime and imipenem. Species level identification was attempted by performing biochemical tests such as casein, L-tyrosine and xanthine hydrolysis and the strain did not show hydrolysis. Based on the biochemical reactions and antibiotic susceptibility pattern, the isolate was identified as Nocardia asteroides.

The patient succumbed a few days later despite the aggressive therapy. Consent for autopsy could not be obtained.

\section{Discussion}

The incidence of nocardiosis has increased over the past three decades and $N$. asteroides is an important opportunistic agent in the immunocompromised hosts. ${ }^{6}$ Immunosuppressive drugs particularly steroids, are known to predispose to the infection. ${ }^{6}$ The extreme rarity of suppurative thyroiditis is attributed to the anatomic isolation of the gland, its rich

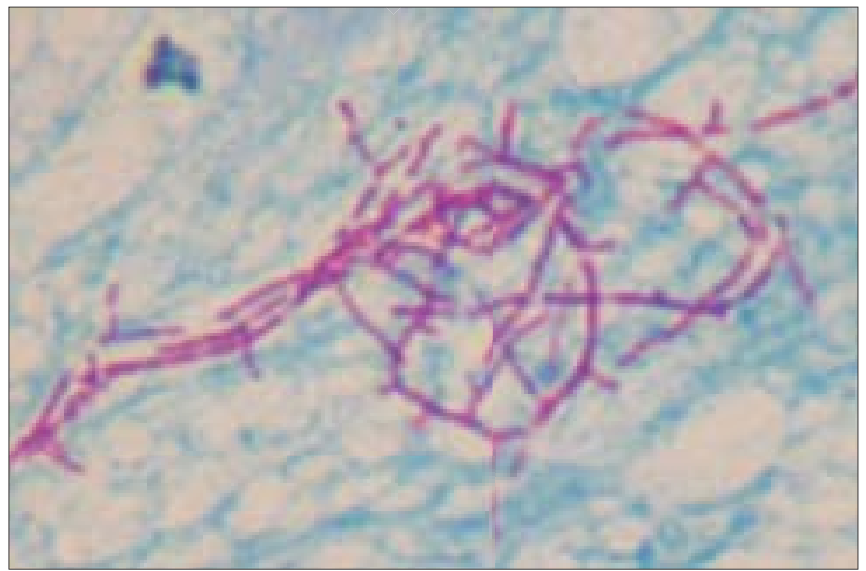

Figure 3: Branching filamentous acidfast bacilli from thyroid tissue (1\% acid fast stain, $\mathrm{x} 1000$ ) system of drainage and the presumptive antibacterial effect of high iodine concentration. ${ }^{3}$ We speculate that the use of corticosteroids in this elderly diabetic patient over a course of many months led to unusual location and the serious clinical manifestation of infection. The delayed diagnosis and institution of therapy, both contributed to the dissemination and death of the patient.

The radiological and clinical features that suggested malignancy were misleading. Therefore, nocardiosis should be always considered in the differential diagnosis of disseminated metastatic lesions particularly in immunosuppressed patients.

Microscopic study of stained smears could direct one to appropriate therapy and cultures provide the etiologic agent. However, it is important to emphasize that the cultures of Nocardia typically take longer than three to five days. Because of this, it is often overlooked in routine cultures. Therefore, one needs to carefully evaluate the Gram smear and the acidfast smear so that these organisms are not missed and early diagnosis is achieved.

Phenotypic tests are inaccurate and cumbersome to identify the species within the Genus Nocardia. ${ }^{8}$ The antibiotic susceptibility testing of Nocardia remains problematic due of lack of standardized validated methods. ${ }^{7}$ There are currently more than 30 species of Nocardia of clinical significance that have variable drug susceptibilities. Correct identification of species is important for clinical management and treatment. Molecular techniques such as PCR restriction enzyme analysis and 16S rRNA gene sequencing have revolutionized the identification of Nocardia. However, this is restricted to referral laboratories. ${ }^{9}$ Our country lacks a referral laboratory where accurate species identification and susceptibility testing can be performed and confirmed. Therefore the routine clinical diagnostic laboratories rely on genus level identification only. This case report suggests that thyroid should be added to the list of tissues that can be the site of infection in patients with disseminated nocardiosis.

\section{Acknowledgement}

We wish to thank Prof. Peerapura and Dr. Shapur, BLDE Medical College, Bijapur, for their help in species identification.

\section{References}

1. Carrier C, Marchandin H, Andrieu JM, Vandome A, Perez C. Nocardia thyroiditis: Unusual location of infection. $J$ Clin Microbiol 1999;37:2323-5.

2. Leong KP, Tee NW, Yap WM, Chee TS, Koh ET. Nocardiosis in patients with systemic lupus erythematosus. The Singapore Lupus Study Group. J Rheumatol 2000;27:1306-12.

3. Vandjme A, Pageaux GP, Bismuth M, Fabre JM, Domergue $\mathrm{J}$, Perez C, et al. Nocardiosis revealed by thyroid abscess 
in a liver-kidney transplant recipient. Transplant Int 2001;14:202-4.

4. Severo CB, Oliveira FM, Cunha L, Cantarelli V, Severo LC. Disseminated nocardiosis due to Nocardia farcinica: Diagnosis by thyroid abscess culture. Rev Inst Med Trop $S$ Paulo 2005;47:355-8.

5. Lewin SR, Street AC, Snider J. Suppurative thyroiditis due to Nocardia asteroides. J Infect 1993;26:339-340

6. Tabrizi SJ. Nocardia pericarditis. BMJ 1994;309:1495-7.

7. Baron EJ, Finegold SM, editors Nocardia, Streptomyces, Rhodococcus, Oerskovia and similar organisms, Chapter
24. In: Bailey and Scott's Diagnostic Microbiology, $11^{\text {th }}$ ed. The CV Mosby Company: St. Louis; 2002. p. 351-62.

8. Kiska DL, Hicks K, Pettit DJ. Identification of medically relevant Nocardia species with an abbreviated battery of tests. J Clin Microbiol 2002;40:1346-51.

9. Brown-Elliott BA, Brown JM, Conville PS, Wallace RJ Jr. Clinical and laboratory features of the Nocardia spp. Based on current molecular taxonomy. Clin Microbiol Rev 2006;19:259-82.

Source of Support: Nil, Conflict of Interest: None declared. 